\section{Änderung der thermischen und elektrischen Leitfähigkeit der ferromagnetischen Stoffe im magnetischen Feld}

\author{
Von Helena Papadimitraki
}

Aus dem Physikalischen Institut der Universität von Thessaloniki

(Z. Naturforschg. 16 a, 217 [1961] ; eingegangen am 14. November 1960)

Zur Messung der Wärmeleitfähigkeit mittels der Methode der stationären Strömung ${ }^{1}$ wurden stabförmige Proben (ca. $5 \mathrm{~mm} \phi$ ) verwandt. Die elektrische Leitfähigkeit wurde an drahtförmigen Proben (ca. $2 \mathrm{~mm} \phi$ ) durch Messung von Stromstärke und Spannungsabfall bestimmt.

Aus den beiden relativen magnetischen Leitfähigkeitsänderungen wird die LoRentzsche Zahl berechnet. Es ergab sich:

A. Für Nickel:

1. Die Wärmeleitfähigkeit des Nickels erniedrigt sich im magnetischen Parallelfeld, sie vergrößert sich im Querfeld. Mit zunehmender Feldstärke steigt sie im Parallelfeld nach Durchlaufen eines Minimums (in etwa $1000 \mathrm{Oe})$ an, ohne jedoch den ursprünglichen Wert wieder zu erreichen. Im Querfeld dagegen wächst die Wärmeleitfähigkeit monoton mit der Feldstärke. Proben verschiedener Reinheit unterscheiden sich nur in quantitativer Hinsicht.

2. Hysteresiserscheinungen werden nur bei schwachen Feldstärken beobachtet.

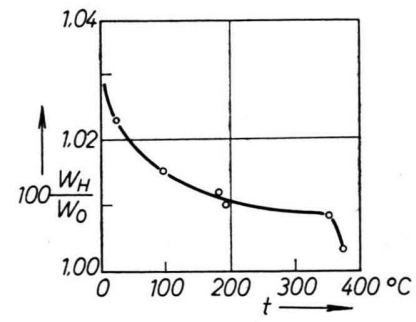

Abb. 1. $W_{\mathrm{H}}$ Wärmewiderstand im Magnetfeld, $W_{0}$ Wärmewiderstand ohne Magnetfeld.

1 Roberts Miller, Heat and Thermodynamic, Bd. IV, S. 278 [1928].

2 B. Swensson, Ann. Phys., Lpz. 22, 97 [1935].

3 E. H. Sondheimer u. B. A. Wilson, Proc. Roy. Soc., Lond. A 203, 75 [1950].
3. Der Einfluß des Magnetfeldes nimmt mit zunehmender Temperatur $a^{2}{ }^{2}$ und schwindet sehr rasch bei Annäherung an die Curie-Temperatur (Abb. 1).

4. Die elektrische Leitfähigkeit zeigt qualitativ dieselben Erscheinungen wie die thermische Leitfähigkeit, aber der Magnetfeldeinfluß ist im elektrischen Fall etwas geringer als im thermischen. Die bis jetzt untersuchten nicht ferromagnetischen Metalle verhalten sich umgekehrt ${ }^{3-5}$.

5. Im Parallelfeld nimmt die Lonentzsche Zahl $L_{\mathrm{H}}$ zunächst mit wachsender Feldstärke ab, um nach Durchlaufen eines Minimums wieder anzusteigen. Ihr Minimalwert beträgt $\mathbf{7 \%} \%$ weniger als der ursprüngliche Wert $L_{0}$ (Abb. 2). In dem untersuchten Feldstärkebe-

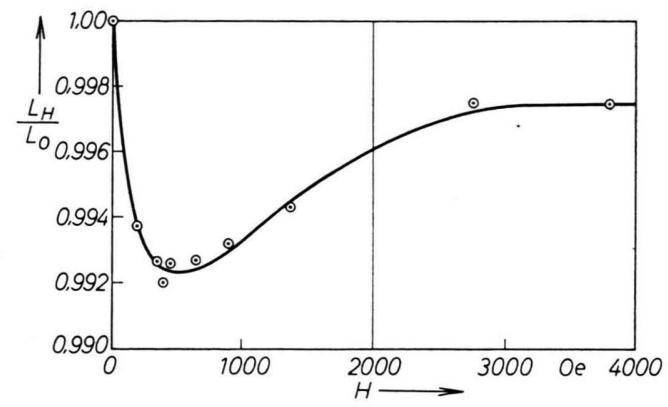

Abb. 2. Die Lorentzsche Zahl in Abhängigkeit von der Stärke des Magnetfeldes $H$.

reich (5000 0e) wird der Ausgangswert nicht wieder erreicht.

6. Im Querfeld läßt sich eine Änderung der LoRentzschen Zahl oberhalb 1000 Oe nachweisen. Bei dieser Feldstärke beginnt die Lorentzsche Zahl mit wachsendem Feld monoton anzusteigen. Bei 9000 Oe wird eine Änderung um 6\% des Ausgangswertes beobachtet.

B. Für Eisen :

Sowohl im Parallelfeld als auch im Querfeld erniedrigt sich die Wärmeleitfähigkeit. Die Effekte sind jedoch sehr viel kleiner als beim Nickel, so daß man keine quantitativen Resultate herausziehen kann ${ }^{6}$.

4 E. Grüneisen, Ann. Phys., Lpz. 32, 219 [1938].

5 E. Grüneisen u. J. Gielessen, Ann. Phys., Lpz. 62, 449 [1936].

6 Brown, Phys. Rev. 32, 508 [1928]. 